\title{
Role of Carbon Nanotubes to Enhance the Long-Term Stability of Dye-Sensitized Solar
}

\section{Cells}

Mahyar Mohammadnezhad ${ }^{\mathrm{a}}$, Gurpreet Singh Selopal ${ }^{\mathrm{a}, \mathrm{b}}{ }^{*}$, Zhiming M. Wang, ${ }^{\mathrm{b}}$ Barry Stansfield a, Haiguang Zhao ${ }^{c^{*}}$, Federico Rosei ${ }^{\mathrm{a}, *}$

${ }^{a}$ Institut National de la Recherche Scientifique, Centre Énergie, Matériaux et Télécommunications 1650 Boul. Lionel Boulet Varennes, Québec, J3X 1S2, Canada.

b Institute of Fundamental and Frontier Sciences, University of Electronic Science and Technology of China

Chengdu 610054, P. R. China.

c College of Physics \& State Key Laboratory of Bio-Fibers and Eco-Textiles, Qingdao University, Qingdao 266071, P. R. China.

Email: gurpreet.selopal@emt.inrs.ca; hgzhao@qdu.edu.cn; rosei@emt.inrs.ca

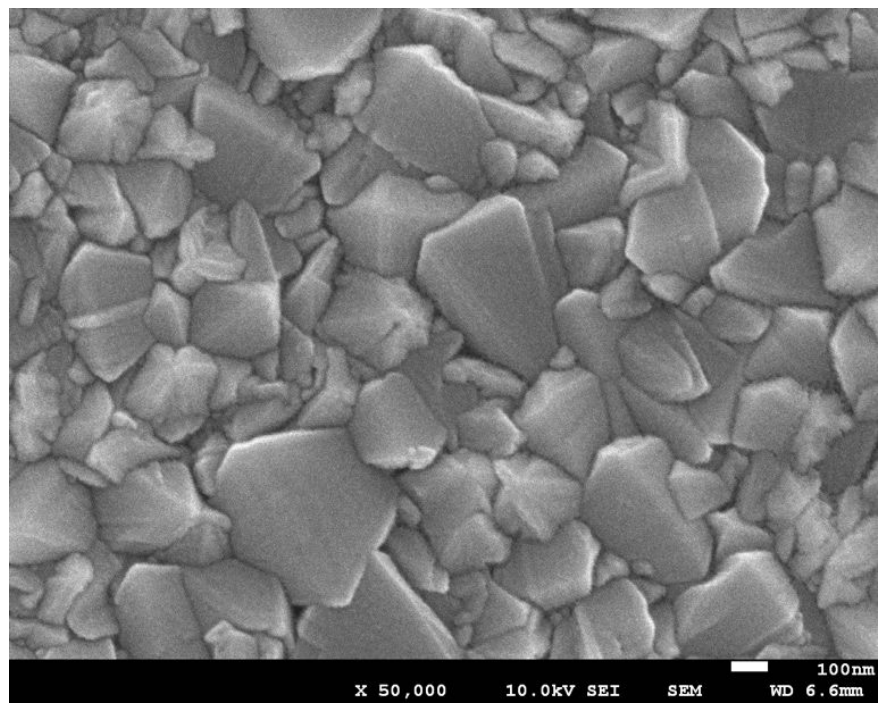

Figure S1 FESEM images of the FTO conducting glass. 


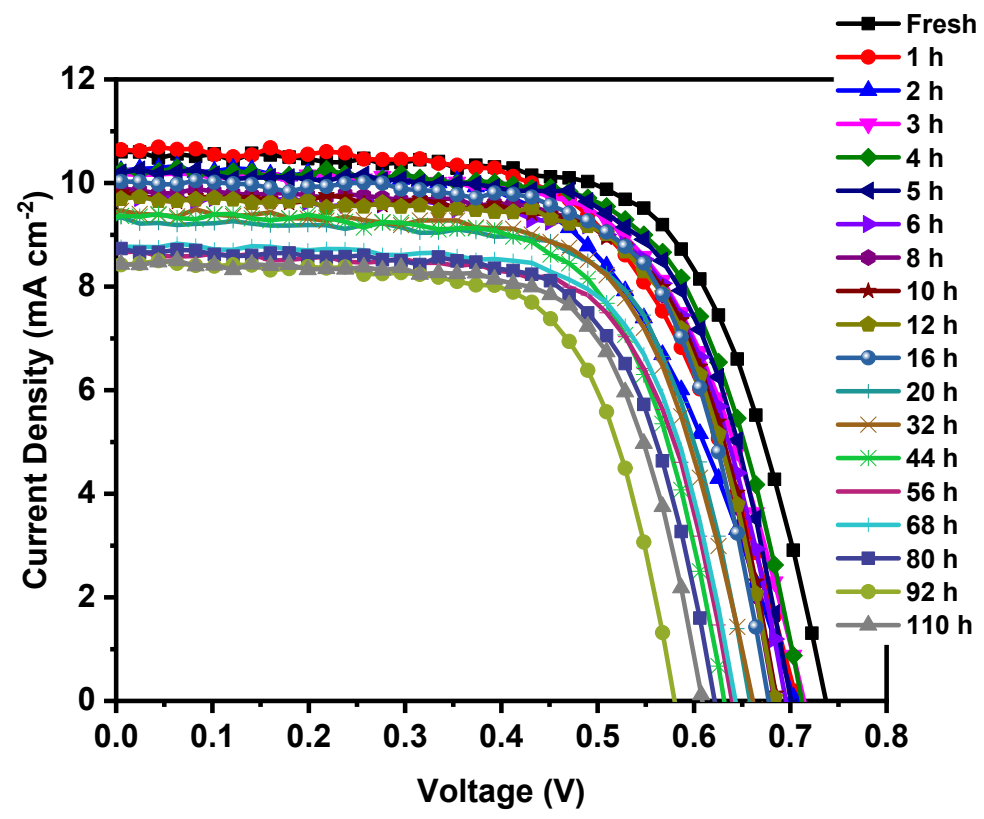

Figure S2 Current density-voltage curve for DSSCs without MWCNTs as a function of soaking time under continuous one sun light irradiation.

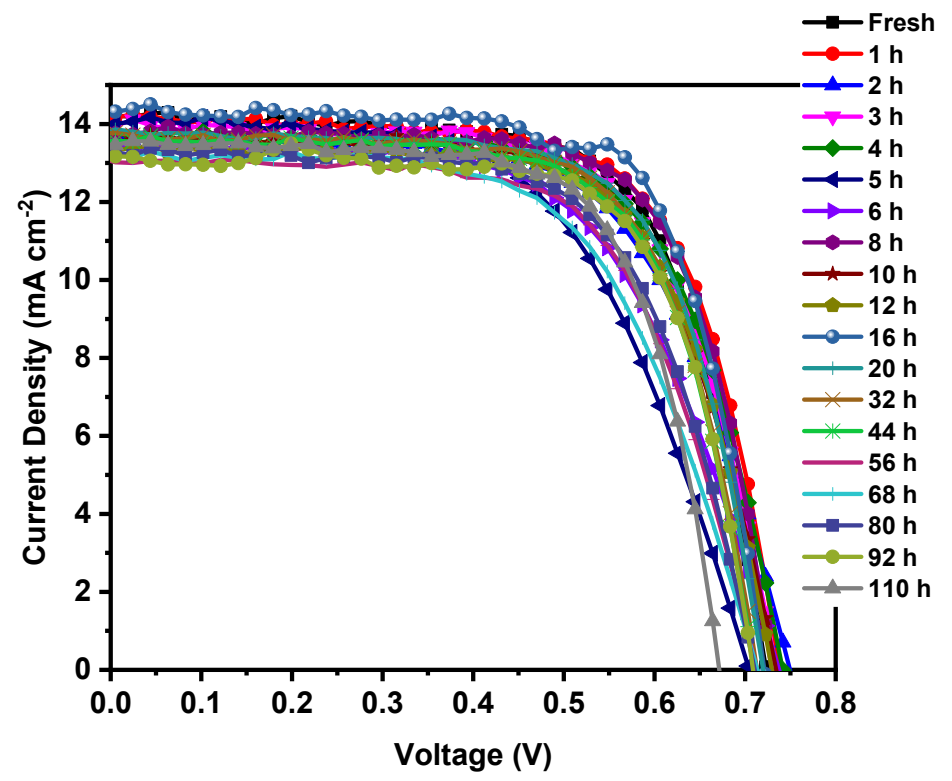

Figure S3 Current density-voltage curve for DSSCs with MWCNTs as a function of soaking time under continuous one sun light irradiation. 


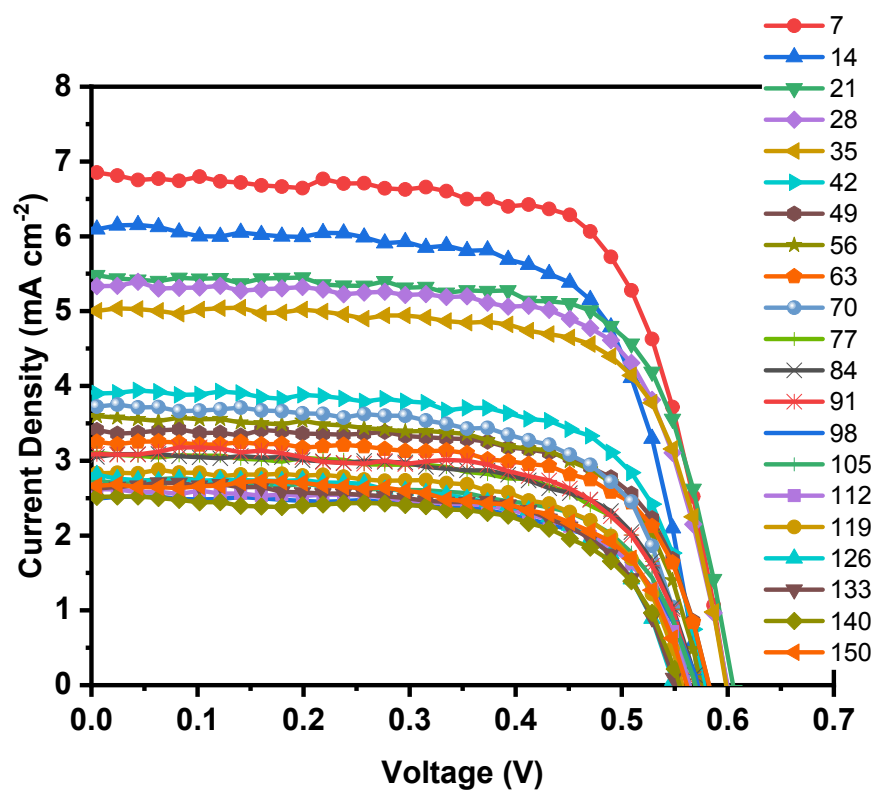

Figure S4 Current density-voltage curve for DSSCs without MWCNTs as a function of soaking time (day) under continuous indoor light.

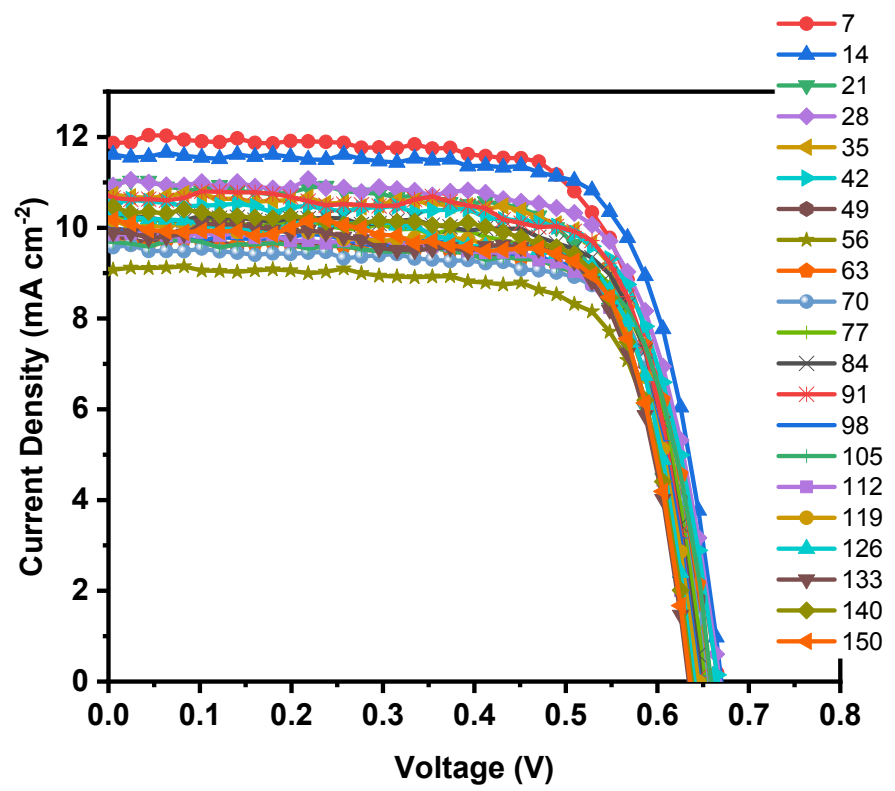

Figure S5 Current density-voltage curve for DSSCs with MWCNTs as a function of soaking time (day) under continuous indoor light. 


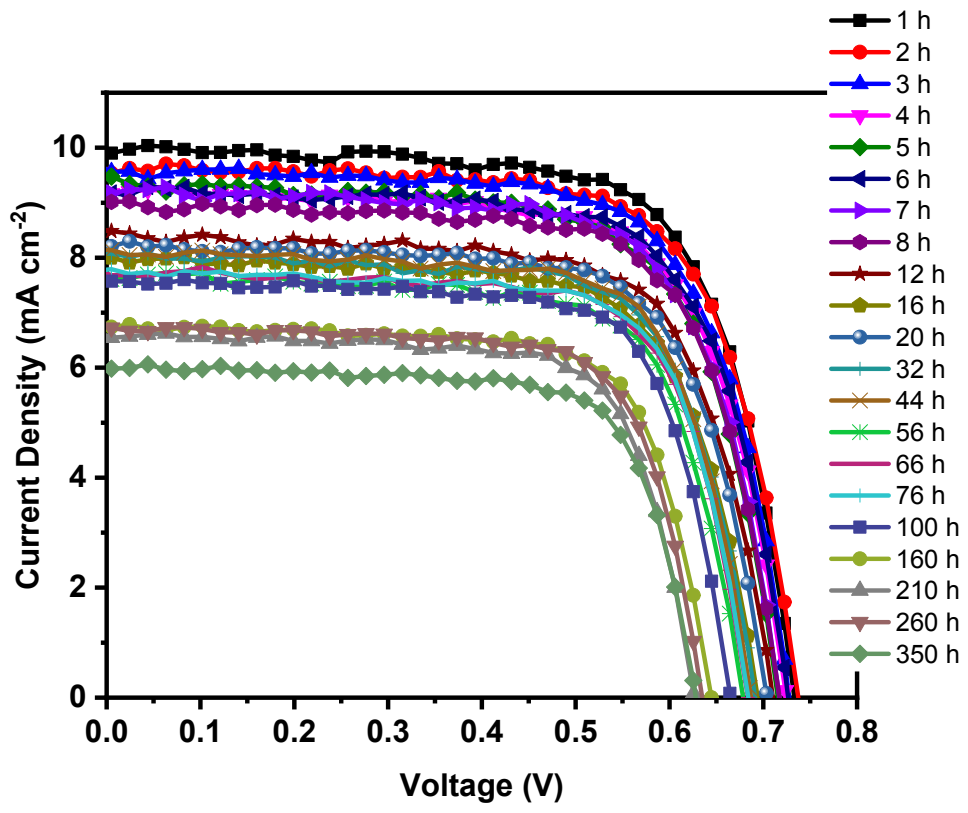

Figure S6 Current density-voltage curve for DSSCs without MWCNTs as a function of soaking time under continuous UV light.

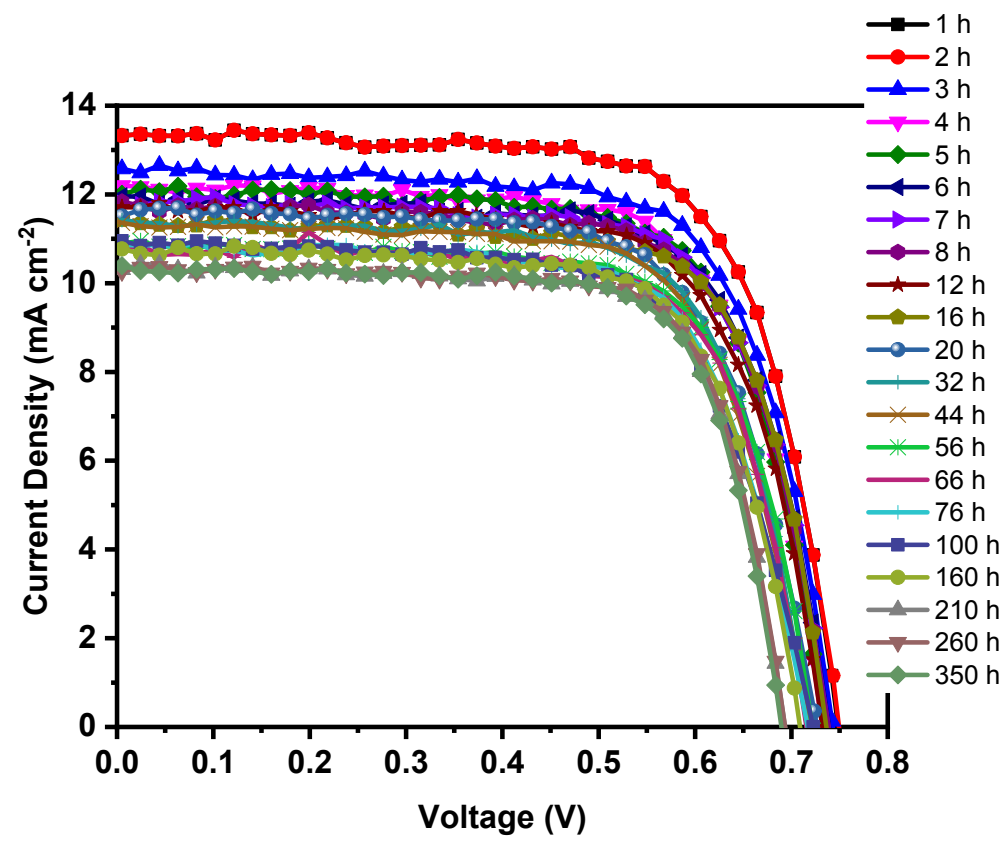

Figure S7 Current density-voltage curve for DSSCs with MWCNTs as a function of soaking time under continuous UV light. 


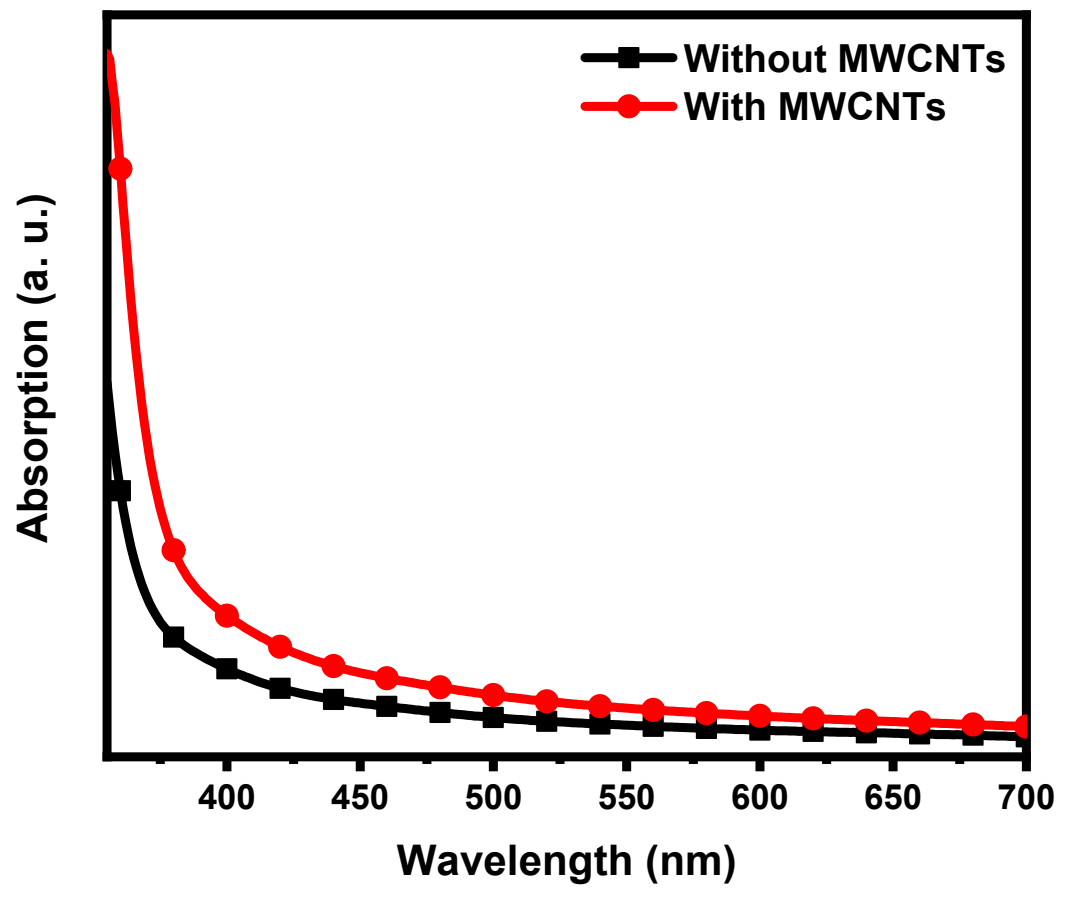

Figure S8 UV-vis absorption spectra of the $\mathrm{TiO}_{2}$ and $\mathrm{TiO}_{2}-\mathrm{MWCNTs}$ films.
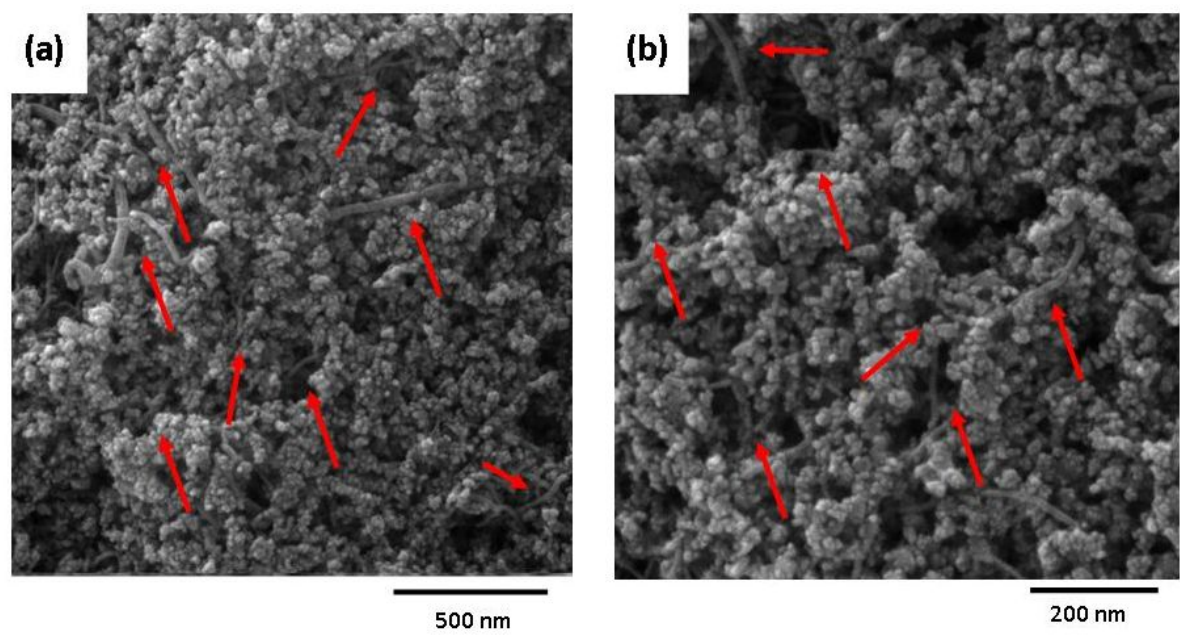

Figure S9 FESEM images of the photoanodes with $0.03 \%$ wt of MWCNTs. Red arrows indicate MWCNTs. 

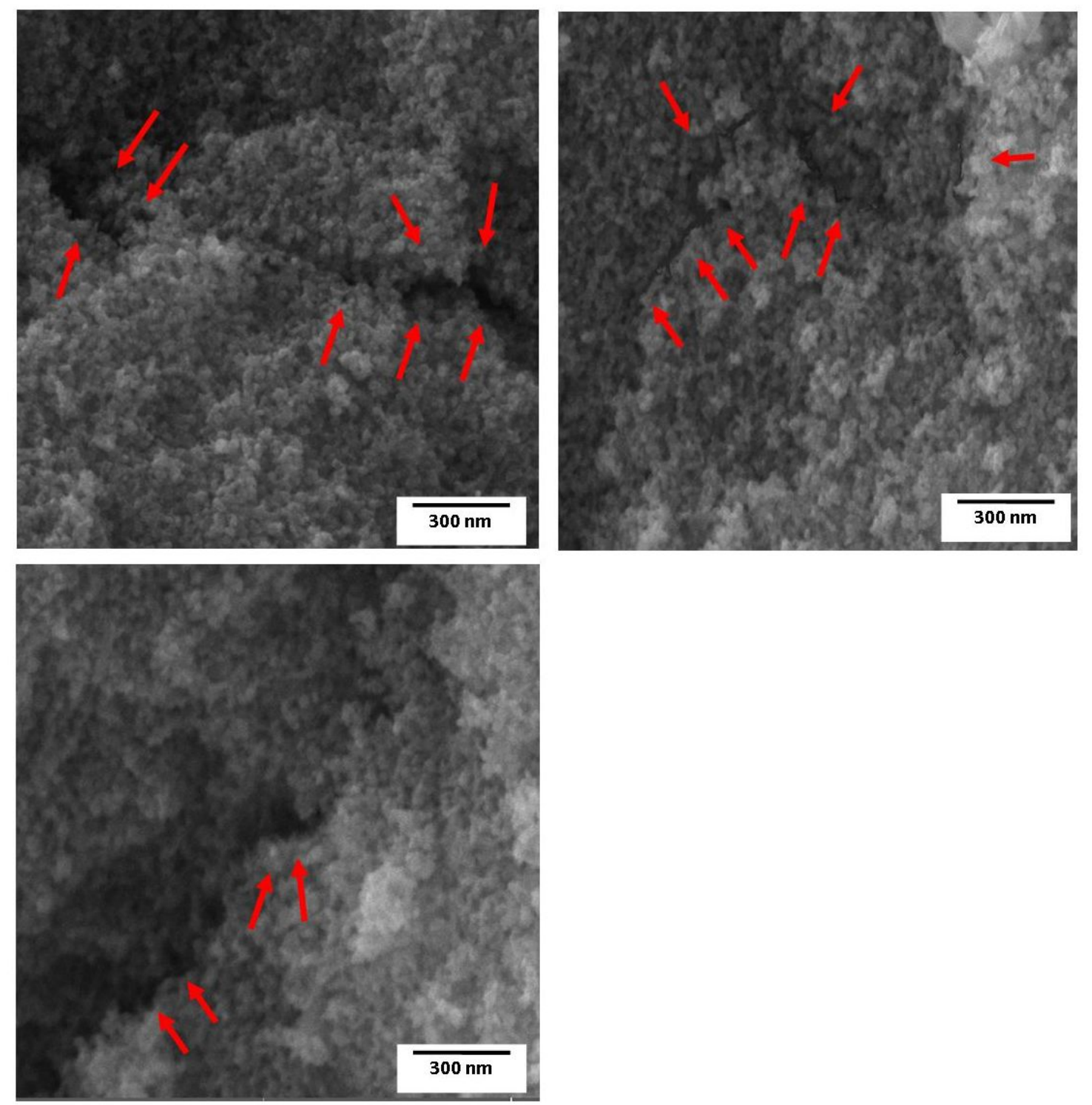

Figure S10 FESEM images of the photoanodes without MWCNTs after $200 \mathrm{~h}$ continuous under one sun light (100 $\left.\mathrm{mW} \mathrm{cm}^{-2}\right)$ irradiation and 70 days storage under continuous indoor light (30 $\mathrm{mW} \mathrm{cm} \mathrm{cm}^{-2}$ ). Red arrows indicate cracks in the photoanode. 

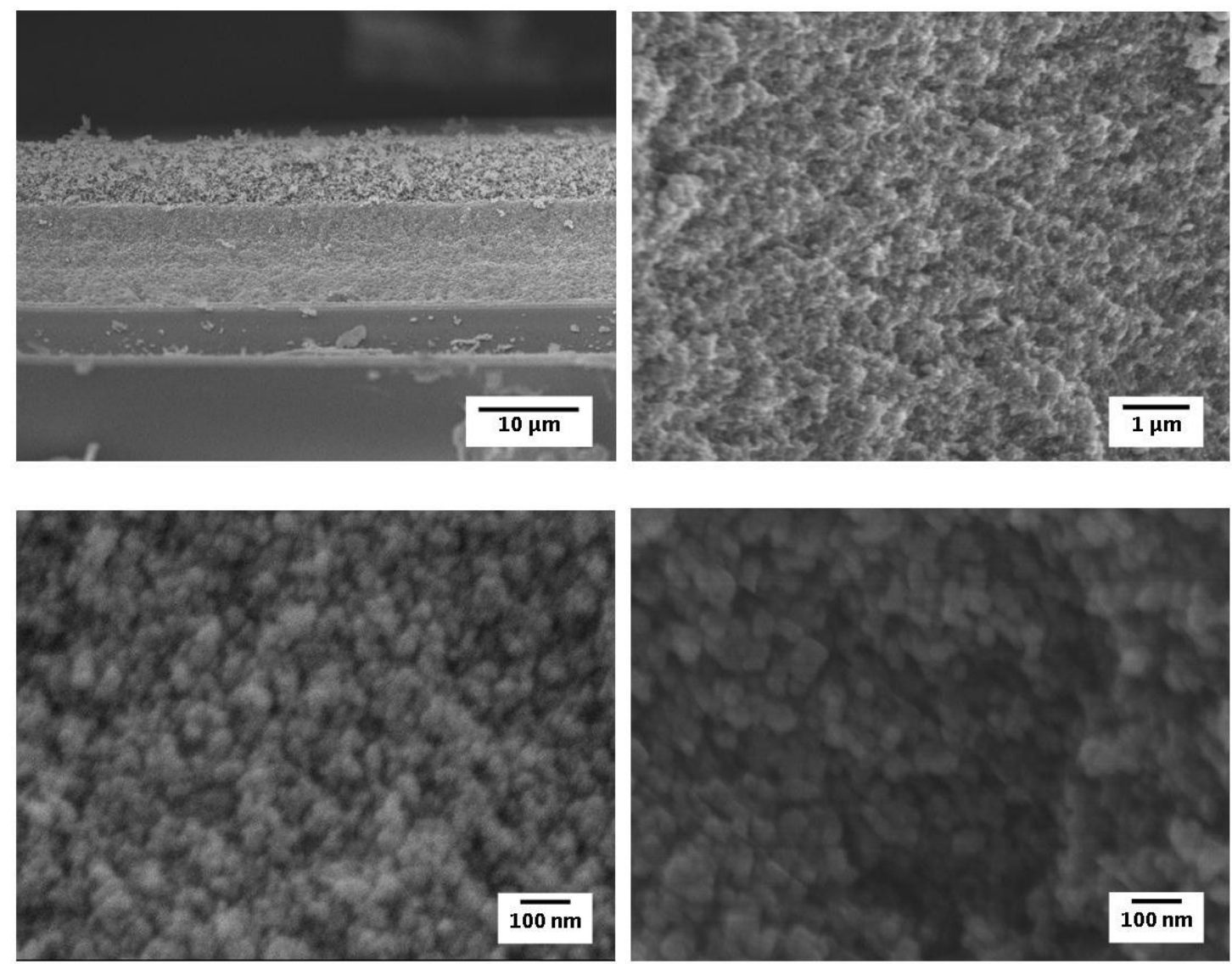

Figure S11 FESEM images of the photoanodes with MWCNTs after $200 \mathrm{~h}$ continuous under one sun light $\left(100 \mathrm{~mW} \mathrm{~cm}^{-2}\right)$ irradiation and 70 days storage under continuous indoor light (30 $\mathrm{mW}$ $\left.\mathrm{cm}^{-2}\right)$. 

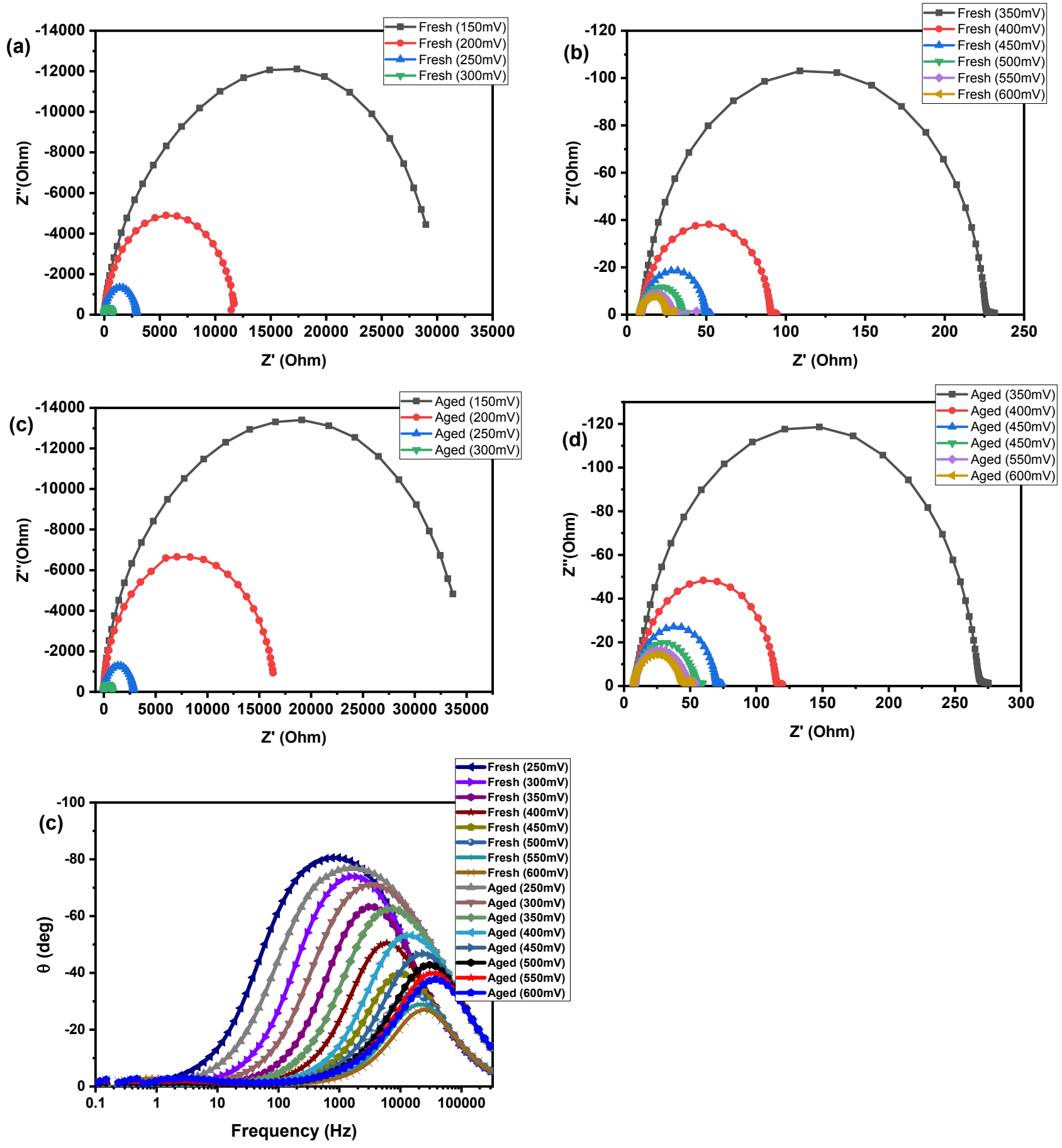

Figure S12 (a-d) Electrochemical impedance spectroscopy analysis of the fresh and aged cells without MWCNTs as a function of applied biases, (e) Bode phase of the fresh and aged cells without MWCNTs. 

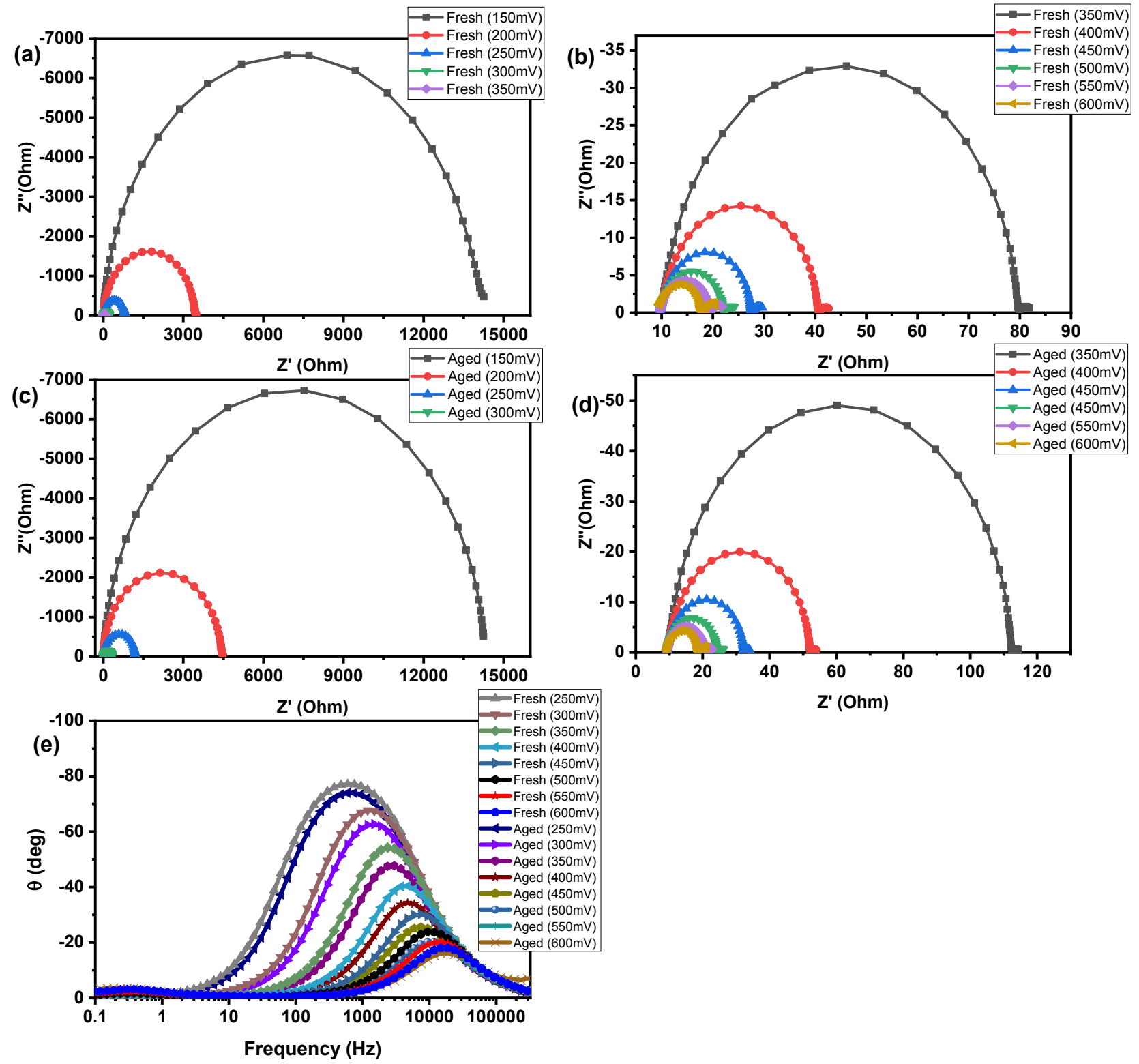

Figure S13 (a-d) Electrochemical impedance spectroscopy analysis of the fresh and aged cells with MWCNTs as a function of applied biases, (e) Bode phase of the fresh and aged cells with MWCNTs. 